\title{
Littlewood problem for a singular subquadratic potential
}

(Dedicated to Professor George R. Sell on the occasion of his 70th birthday)

Xiong $\mathrm{Li}^{a, *, 1}$, Yingfei $\mathrm{Yi}^{b, 2}$

${ }^{a}$ School of Mathematical Sciences, Beijing Normal University, Beijing 100875, P R China

${ }^{b}$ School of Mathematics, Georgia Institute of Technology, Atlanta, GA 30332, USA

\begin{abstract}
We consider a periodically forced singular oscillator in which the potential has subquadratic growth at infinity and admits a singularity. Using Moser's twist theorem of invariant curves, we show the existence of quasi-periodic solutions. This solves the Littlewood problem on the boundedness of all solutions for such a system.

Keywords: Littlewood problem; boundedness of solutions; quasi-periodic solutions; Moser's twist theorem
\end{abstract}

MSC: $34 \mathrm{C} 15,58 \mathrm{~F} 27$

\section{Introduction}

In this paper, we will consider the Littlewood problem for the forced nonlinear oscillator

$$
\ddot{x}+V^{\prime}(x)=e(t),
$$

where $e(t)$ is an 1-periodic continuous function.

In the early 1960's, Littlewood [5] suggested to study the boundedness of all solutions of systems like (1.1) for the following two cases:

(1) Superlinear case: $\frac{V^{\prime}(x)}{x} \rightarrow+\infty$ as $x \rightarrow \pm \infty$;

(2) Sublinear case: $\operatorname{sign}(x) V^{\prime}(x) \rightarrow+\infty$ and $\frac{V^{\prime}(x)}{x} \rightarrow 0$ as $x \rightarrow \pm \infty$.

The first result in the superlinear case is due to Morris [11], who proved the boundedness of all solutions of (1.1) for $V(x)=\frac{1}{2} x^{4}$. Later, Dieckerhoff and Zehnder [1] considered the polynomial case

$$
\ddot{x}+x^{2 n+1}+\sum_{i=0}^{2 n} p_{i}(t) x^{i}=0,
$$

where $p_{i}, i=0,1, \cdots, 2 n$, are 1-periodic $C^{\infty}$ functions, and showed the boundedness of all solutions. Subsequently, this result was extended to more general cases by several authors (see [3], [4], [6], [16], [17] and references therein).

\footnotetext{
* Corresponding author. E-mail addresses: xli@bnu.edu.cn (X. Li), yi@math.gatech.edu (Y. Yi)

1 Partially supported by the Program for New Century Excellent Talents of Ministry of Education of China and NSFC Grant 10671020.

${ }^{2}$ Partially supported by NSF grant DMS0708331, NSFC Grant 10428101, and a Changjiang Scholarship from Jilin University.
} 
Recently, the boundedness of all solutions was shown by Küpper and You [2] for the sublinear equation

$$
\ddot{x}+|x|^{\alpha-1} x=e(t),
$$

where $0<\alpha<1$ and $e$ is smooth. The general sublinear case was later considered by Liu [7] under certain reasonable conditions.

The Littlewood problem for singular potentials is known to be challenging and there are only very a few results. A case of such oscillators with semilinearly growing potential was recently considered by Capietto-Dambrosio-Liu [8] for which the boundedness of all solutions was shown under some nonresonance conditions (see also Liu [9] for an extension of the result to the isochronous case).

The present paper is devoted to the study of Littlewood problem for forced oscillators with singular sublinearly growing potential. While our result and technique hold for general potentials of the like, we will consider a model problem of (1.1) with

$$
V(x)=x_{+}^{\alpha+1}+\frac{1}{1-x_{-}^{2}}-1= \begin{cases}x^{\alpha+1}, & \text { if } \quad x \geq 0 ; \\ \frac{1}{1-x^{2}}-1, & \text { if }-1<x<0,\end{cases}
$$

where $\frac{1}{3}<\alpha<1, x_{+}=\max \{x, 0\}, x_{-}=\max \{-x, 0\}$.

The main result in this paper is the following

Theorem. Consider the forced oscillator (1.1) with the singular potential (1.3) and $e(t) \in$ $C^{4}(\mathbb{R} / \mathbb{Z})$. Then any solution $x(t)$ of the oscillator with the initial value $x(0)>-1$ satisfies

$$
\inf _{t \in \mathbb{R}} x(t)>-1, \quad \sup _{t \in \mathbb{R}}(|x(t)|+|\dot{x}(t)|)<+\infty .
$$

The power range $\frac{1}{3}<\alpha<1$ and the smoothness requirement of $e(t)$ in the Theorem are due to the technique we employee. We do not know whether they are essential to the validity of the theorem.

The proof of Theorem concerns the formal reduction to normal form and estimates. The formal reduction consists of the following sequence of transformations:

$$
\begin{gathered}
\left(x, \dot{x}, t ; \mathcal{H}=\frac{\dot{x}^{2}}{2}+V(x)+y E(t)\right) \stackrel{A}{\longrightarrow}\left(\theta, I, t ; H=H_{0}(I)+H_{1}(\theta, I, t)\right) \\
\stackrel{B}{\longrightarrow}\left(t, H, \theta ; I=I_{0}(H)+I_{1}(t, H, \theta)\right),
\end{gathered}
$$

where $E(t)=\int_{0}^{t} e(s) d s$, in which $A$ is the standard action-angle reduction of $(x, \dot{x})$ into $(\theta, I)$, and $B$ is the change of time and energy into the new position and momentum with the angle $\theta$ playing the role of new time. For each step of transformations, detailed estimates will be given. In particular, due to the existence of singularity in the potential, special cares are needed on estimates with respect to $H_{0}(I), x(\theta, I)$, and $y(\theta, I)$, for which we will employee some techniques developed in [3], [7] and [8]. An application of Moser's twist theorem will then yield the Theorem.

The rest of the paper is organized as follows. In Sections 2, 3, we introduce transformations $A, B$ respectively, along with some technical estimates. The Theorem will be proved in 
Section 4. We will also give another result on the existence of quasi-periodic solutions, Aubry-Mather sets, and unlinked periodic solutions in this section. The Appendix is devoted to the estimates on $I_{0}(H), x(\theta, I)$, and $y(\theta, I)$.

For simplicity, throughout the paper we use symbols $c, C$ to denote appropriate universal constants which are in $(0,1),(1, \infty)$ respectively.

\section{Action-angle variables}

Without loss of generality, we assume that the average value of $e(t)$ vanishes, i.e., the function

$$
E(t)=\int_{0}^{t} e(s) d s
$$

is 1-periodic and of class $C^{5}$. Indeed, if $\bar{e}=\int_{0}^{1} e(t) \neq 0$, then we can use

$$
\tilde{V}(x)= \begin{cases}x^{\alpha+1}-\bar{e} x, & \text { if } \quad x \geq 0 ; \\ \frac{1}{1-x^{2}}-1, & \text { if }-1<x<0,\end{cases}
$$

and $\tilde{e}(t)=e(t)-\bar{e}$ in place of $V(x)$ and $e(t)$ respectively in (1.1).

It is clear that the oscillator (1.1) is a Hamiltonian system of one-and-a-half degree of freedom:

$$
\dot{x}=\partial_{y} \mathcal{H}, \quad \dot{y}=-\partial_{x} \mathcal{H},
$$

where

$$
\mathcal{H}(x, y, t)=\frac{1}{2} y^{2}+V(x)+y E(t) .
$$

For each $H>0$, we denote by $I_{0}(H)$ the area enclosed by the closed curve

$$
\Gamma_{H}: \frac{1}{2} y^{2}+V(x)=H .
$$

Let $-1<-\alpha_{H}<0<\beta_{H}$ be such that

$$
V\left(-\alpha_{H}\right)=V\left(\beta_{H}\right)=H,
$$

i.e.,

$$
\alpha_{H}=\sqrt{\frac{H}{H+1}}, \quad \beta_{H}=H^{\frac{1}{1+\alpha}} .
$$

It is easy to see that

$$
I_{0}(H)=2 \int_{-\alpha_{H}}^{\beta_{H}} \sqrt{2(H-V(\xi))} d \xi
$$

and

$$
T_{0}(H):=I_{0}^{\prime}(H)=2 \int_{-\alpha_{H}}^{\beta_{H}} \frac{d \xi}{\sqrt{2(H-V(\xi))}} .
$$

Denote

$$
\begin{array}{ll}
I_{0}^{+}(H)=2 \int_{0}^{\beta_{H}} \sqrt{2(H-V(\xi))} d \xi, & I_{0}^{-}(H)=2 \int_{-\alpha_{H}}^{0} \sqrt{2(H-V(\xi))} d \xi \\
T_{0}^{+}(H)=2 \int_{0}^{\beta_{H}} \frac{d \xi}{\sqrt{2(H-V(\xi))}}, & T_{0}^{-}(H)=2 \int_{-\alpha_{H}}^{0} \frac{d \xi}{\sqrt{2(H-V(\xi))}} .
\end{array}
$$


Then

$$
I_{0}(H)=I_{0}^{+}(H)+I_{0}^{-}(H), \quad T_{0}(H)=T_{0}^{+}(H)+T_{0}^{-}(H) .
$$

Now, the action-angle variable $(I, \theta), I>0, \theta \in \mathbb{R}(\bmod 1)$, can be introduced as usual:

$$
I=I_{0}(H(x, y)),
$$

and

$$
\theta=\theta(x, y)= \begin{cases}\frac{1}{T_{0}(H(x, y))}\left(\int_{-\alpha_{H}}^{x} \frac{d \xi}{\sqrt{2(H(x, y)-V(\xi))}}\right), & \text { if } y>0 \\ 1-\frac{1}{T_{0}(H(x, y))}\left(\int_{-\alpha_{H}}^{x} \frac{d \xi}{\sqrt{2(H(x, y)-V(\xi))}}\right), & \text { if } y<0\end{cases}
$$

for $(x, y) \in(-1,+\infty) \times \mathbb{R} \backslash\{(0,0)\}$, where

$$
H(x, y)=\frac{1}{2} y^{2}+V(x) .
$$

Also we let $x(\theta, I), y(\theta, I)$ be inverse functions of (2.2) and (2.3).

With the action-angle variable $(I, \theta)$, system $(2.1)$ becomes

$$
\dot{I}=-\partial_{\theta} H, \quad \dot{\theta}=\partial_{I} H,
$$

where

$$
H=H(\theta, I, t)=H_{0}(I)+H_{1}(\theta, I, t),
$$

in which $H_{0}(I)$ is the inverse function of $I_{0}(H)$ and

$$
H_{1}(\theta, I, t)=y(\theta, I) E(t) .
$$

We now give some estimates on the functions $H_{0}(I), H_{1}(\theta, I, t), x(\theta, I)$, and $y(\theta, I)$.

Lemma 2.1 The following holds:

(1) $\sqrt{2} H^{\frac{1}{2}+\frac{1}{1+\alpha}} \leq I_{0}^{+}(H) \leq 2 \sqrt{2} H^{\frac{1}{2}+\frac{1}{1+\alpha}}$;

(2) $\sqrt{2} H^{\frac{1}{2}}\left(\frac{H}{H+1}\right)^{\frac{1}{2}} \leq I_{0}^{-}(H) \leq 2 \sqrt{2} H^{\frac{1}{2}}\left(\frac{H}{H+1}\right)^{\frac{1}{2}}$;

(3) $T_{0}^{+}(H)=\frac{d I_{0}^{+}(H)}{d H}=\left(\frac{1}{2}+\frac{1}{1+\alpha}\right) H^{-1} I_{0}^{+}(H)$;

(4) $\frac{d T_{0}^{+}(H)}{d H}=\frac{d^{2} I_{0}^{+}(H)}{d H^{2}}=\left(\frac{1}{(1+\alpha)^{2}}-\frac{1}{4}\right) H^{-2} I_{0}^{+}(H)$.

Proof (1) (resp. (2)) can be easily proved by comparing the area bounded by $\Gamma_{H}$ in the right half plane (resp. the left half plane) with the area of the triangle or rectangle with sides $\sqrt{2 H}$ and $\beta_{H}\left(\right.$ resp. $\left.\alpha_{H}\right)$.

Similar to the proof of $[3, \mathrm{~A} 3.2]$, we have

$$
\begin{aligned}
T_{0}^{+}(H) & =\frac{d I_{0}^{+}(H)}{d H}=\frac{2}{H} \int_{0}^{\beta_{H}}\left(\frac{1}{2}+W^{\prime}(\xi)\right) \sqrt{2(H-V(\xi))} d \xi \\
& =\frac{2}{H}\left(\frac{1}{2}+\frac{1}{1+\alpha}\right) \int_{0}^{\beta_{H}} \sqrt{2(H-V(\xi))} d \xi=\left(\frac{1}{2}+\frac{1}{1+\alpha}\right) \frac{I_{0}^{+}(H)}{H}
\end{aligned}
$$


with $W(x)=\frac{V(x)}{V^{\prime}(x)}$. This proves $(3)$.

(4) easily follows from (3).

Lemma 2.2 For all $n=0,1, \cdots$,

$$
\frac{d^{n} T_{0}^{-}(H)}{d H^{n}}=(-1)^{n} \frac{(2 n-1) ! !}{2^{n}} \frac{\sqrt{2}}{H^{(2 n+1) / 2}}+o\left(\frac{1}{H^{(2 n+1) / 2}}\right)
$$

as $H \rightarrow+\infty$. Consequently,

$$
\left|\frac{d^{n} I_{0}^{-}(H)}{d H^{n}}\right| \leq C H^{-n} I_{0}^{-}(H)
$$

for all $n \geq 0$ as $H \gg 1$.

Proof This lemma was proved in [8]. For the readers' convenience, we include the proof in the Appendix.

Remark 2.1 (1). It follows from the Lemmas 2.1, 2.2 that

$$
\lim _{H \rightarrow+\infty} T_{0}^{+}(H)=+\infty, \quad \lim _{H \rightarrow+\infty} T_{0}^{-}(H)=0 .
$$

Thus the time period $T_{0}(H)$ of the integral curve $\Gamma_{H}$ is dominated by $T_{0}^{+}(H)$ when $H$ is sufficiently large.

(2). It also follows from Lemmas 2.1, 2.2 that

$$
c H^{-n} I_{0}(H) \leq\left|\frac{d^{n} I_{0}(H)}{d H^{n}}\right| \leq C H^{-n} I_{0}(H)
$$

for all $n=0,1, \cdots$ as $H$ sufficiently large. In particular,

$$
c H^{\frac{1}{2}+\frac{1}{1+\alpha}} \leq I_{0}(H) \leq C H^{\frac{1}{2}+\frac{1}{1+\alpha}}
$$

as $H$ sufficiently large.

(3). Note that $H_{0}(I)$ is the inverse function of $I_{0}(H)$. We have by (2.6) and (2.7) that

$$
\begin{aligned}
& \left|\frac{d^{n} H_{0}(I)}{d I^{n}}\right| \leq C I^{-n} H_{0}(I), \\
& c I^{\frac{3+\alpha}{2+2 \alpha}} \leq H_{0}(I) \leq C I^{\frac{3+\alpha}{2+2 \alpha}}
\end{aligned}
$$

for all $n=0,1, \cdots$ as I sufficiently large.

Lemma 2.3 As $x>-1$ and I sufficiently large,

$$
\left|\partial_{I}^{n} x(\theta, I)\right| \leq C I^{-n}|x(\theta, I)+1|, \quad\left|\partial_{I}^{n} y(\theta, I)\right| \leq C I^{-n}|y(\theta, I)|
$$

for all $n=0,1, \cdots$.

Proof See Appendix.

Lemma 2.4 As I sufficiently large,

$$
\left|I^{k} \partial_{I}^{k} \partial_{t}^{l} H_{1}(\theta, I, t)\right| \leq C \sqrt{H_{0}(I)}, \quad 0 \leq k+l \leq 5 .
$$

Proof It follows from the definition of $H_{1}(\theta, I, t)$, Lemma $2.1(2)$, and the fact that $|y(\theta, I)| \leq$ $\sqrt{2 H_{0}(I)}$. 


\section{New action-angle variables}

Now we consider the forced Hamiltonian $H(\theta, I, t)$ in $(2.5)$. The identity

$$
I d \theta-H(\theta, I, t) d t=-(H d t-I(t, H, \theta) d \theta)
$$

implies that if one can solve $I=I(t, H, \theta)$ in $H$ from (2.5) with $\theta$ and $t$ as parameters, then the Hamiltonian system (2.4) becomes

$$
\frac{d t}{d \theta}=\partial_{H} I(t, H, \theta), \quad \frac{d H}{d \theta}=-\partial_{t} I(t, H, \theta)
$$

with Hamiltonian $I(t, H, \theta)$ and new action-angle variables $H, t$, and time variable $\theta$. This point of view has already been adopted in [3].

As $I$ sufficiently, because $\partial_{I} H(\theta, I, t) \neq 0$, one can indeed solve $I(t, H, \theta)$ as the inverse function of $H(\theta, I, t)$ with $t, \theta$ playing the role of parameters. Hence (3.1) is well defined when $H$ is sufficiently large. We define $I_{1}(t, H, \theta)$ as such that

$$
I(t, H, \theta)=I_{0}(H)+I_{1}(t, H, \theta)
$$

Lemma 3.1 As H sufficiently large,

$$
\left|\partial_{H}^{k} \partial_{t}^{l} I_{1}(t, H, \theta)\right| \leq C H^{-k-\frac{1}{2}} I_{0}(H), \quad 0 \leq k+l \leq 5 .
$$

Proof There are three cases to consider. Below, for the sake of brevity, we suspend the $\theta, t$-dependence in most terms.

Case 1: $l=0, k=0$. From the definition of $I(t, H, \theta)$, we have

$$
H_{0}(I(H))+H_{1}(I(H))=H
$$

or equivalently,

$$
I(H)=H_{0}^{-1}\left(H-H_{1}(I(H))\right)=I_{0}\left(H-H_{1}(I(H))\right) .
$$

Denote $H_{1}=H_{1}(I(H))$. Then

$$
\begin{aligned}
I_{1}(H) & =I(H)-I_{0}(H)=I_{0}\left(H-H_{1}(I(H))\right)-I_{0}(H) \\
& =-I_{0}^{\prime}(H) H_{1}+\int_{0}^{H_{1}} s I_{0}^{\prime \prime}\left(H-H_{1}+s\right) d s .
\end{aligned}
$$

We will estimate $I_{1}(H)$ through that of $H_{1}(I(H))$. By Lemmas 2.1, 2.4, and Remark 2.1, we have that $I(H) \rightarrow \infty$ as $H \rightarrow \infty$ and $\left|H_{1}(I)\right|<\frac{1}{2} H_{0}(I)$ for all $\theta, t$ as $I \gg 1$. It follows that

$$
\left|H_{1}(I(H))\right|<\frac{1}{2} H_{0}(I(H))
$$

for all $t, \theta$ as $H \gg 1$. Using (3.3), (3.6) and the monotonicity of $I_{0}$ in $H$, we have

$$
I_{0}\left(\frac{1}{2} H\right)<I(H)<I_{0}\left(\frac{3}{2} H\right)
$$

It follows from Remark 2.1 that 


$$
I_{0}\left(\frac{1}{2} H\right)>c I_{0}(H), \quad I_{0}\left(\frac{3}{2} H\right)<C I_{0}(H),
$$

which implies that

$$
c I_{0}(H)<I(H)<C I_{0}(H) .
$$

Using Remark 2.1 and Lemma 2.4 again, we obtain

$$
\left|H_{1}(I(H))\right| \leq C \sqrt{H_{0}(I(H))} \leq C \sqrt{H_{0}\left(C I_{0}(H)\right)} \leq C \sqrt{H} .
$$

This gives the desired estimate for the first term of (3.5) as

$$
\left|I_{0}^{\prime}(H) H_{1}(I(H))\right| \leq C H^{-\frac{1}{2}} I_{0}(H) .
$$

The second term of (3.5) is bounded by

$$
H_{1}^{2} \sup _{H-H_{1} \leq \widetilde{H} \leq H} I_{0}^{\prime \prime}(\widetilde{H}) \leq C H_{1}^{2} H^{-2} I_{0}(H) \leq C H^{-1} I_{0}(H) .
$$

This completes Case 1.

Case 2: $l=0, k \geq 1$. Differentiating (3.5) $k$ times with respect to $H$ yields

$$
\partial_{H}^{k} I_{1}(H)=-\sum_{i=0}^{k} C_{k i} \partial_{H}^{i+1} I_{0}(H) \partial_{H}^{k-i} H_{1}+\partial_{H}^{k} \int_{0}^{H_{1}} s I_{0}^{\prime \prime}\left(H-H_{1}+s\right) d s,
$$

where $H_{1}=H_{1}(I(H))$ and, for each $i, C_{k i}$ is an integer which only depends on $k$ and $i$. Since

$$
\partial_{H}^{k} H_{1}(I(H))=\sum_{k_{1}+\cdots+k_{j}=k} C_{k j} \partial_{I}^{j} H_{1}(I(H)) \partial_{H}^{k_{1}} I(H) \cdots \partial_{H}^{k_{j}} I(H)
$$

and

$$
\begin{aligned}
& \partial_{H}^{k} \int_{0}^{H_{1}} s I_{0}^{\prime \prime}\left(H-H_{1}+s\right) d s \\
= & \sum_{i, j=0}^{k} C_{k j i} \partial_{H}^{j} H_{1} \partial_{H}^{i} H_{1} \partial_{H}^{k-i-j+2} I_{0}(H) \\
& +\sum_{l_{1}+\cdots+l_{j}=k} C_{k j l} \partial_{H}^{l_{1}} H_{0} \cdots \partial_{H}^{l_{j}} H_{0} \int_{0}^{H_{1}} s \partial_{H}^{j+2} I_{0}\left(H-H_{1}+s\right) d s,
\end{aligned}
$$

the proof of Case 2 is reduced to that of

$$
\left|\partial_{H}^{k} I(H)\right| \leq C H^{-k} I(H), \quad H \gg 1,1 \leq k \leq 5 .
$$

Differentiating (3.2) with respect to $H$ yields

$$
I^{\prime}(H)=I_{0}^{\prime}\left(H-H_{1}\right)\left[1-H_{1}^{\prime} I^{\prime}(H)\right]=\frac{I_{0}^{\prime}\left(H-H_{1}\right)}{1+I_{0}^{\prime}\left(H-H_{1}\right) H_{1}^{\prime}} .
$$

As $H$ sufficiently large, the denominator of the above is close to one and we thus have

$$
I^{\prime}(H)<2 I_{0}^{\prime}\left(H-H_{1}\right)<C\left(H-H_{1}\right)^{-1} I_{0}\left(H-H_{1}\right)<C H^{-1} I(H)
$$


i.e., (3.7) holds when $k=1$.

Using induction, we now assume that for some $n<5$, (3.7) holds for all $1 \leq k \leq n$. Differentiating (3.8) $n$ times with respect to $H$ yields

$$
\partial_{H}^{n+1} I(H)=\sum_{i=0}^{n} C_{n i} \partial_{H}^{i} I_{0}^{\prime}\left(H-H_{1}\right) \partial_{H}^{n-i} \Delta
$$

where $\Delta=\left[1+I_{0}^{\prime}\left(H-H_{1}\right) H_{1}^{\prime}\right]^{-1}$. Hence the proof of $(3.7)$ for $k=n+1$ is reduced to the that of

$$
\begin{aligned}
& \left|\partial_{H}^{m} I_{0}^{\prime}\left(H-H_{1}\right)\right| \leq C H^{-m} I_{0}^{\prime}\left(H-H_{1}\right), \\
& \left|\partial_{H}^{m} \Delta\right| \leq C H^{-m}
\end{aligned}
$$

for all $m \leq n$ and $H \gg 1$.

Since

$$
\begin{aligned}
\partial_{H}^{m} I_{0}^{\prime}\left(H-H_{1}\right) & =\sum_{m_{1}+\cdots+m_{j}=m} C_{m j} I_{0}^{(j+1)}\left(H-H_{1}\right) \partial_{H}^{m_{1}}\left(H-H_{1}\right) \cdots \partial_{H}^{m_{j}}\left(H-H_{1}\right), \\
\partial_{H}^{l}\left(H-H_{1}\right) & =\partial_{H}^{l} H_{0}(I(H))=\sum_{l_{1}+\cdots+l_{i}=l} C_{l i} \partial_{I}^{i} H_{0}(I(H)) \partial_{H}^{l_{1}} I(H) \cdots \partial_{H}^{l_{i}} I(H),
\end{aligned}
$$

the proof of (3.9) easily follows from the induction hypothesis and Remark 2.1.

Note that

$$
\begin{aligned}
\partial_{H}^{m} \Delta= & \sum C_{m j}\left[1+I_{0}^{\prime}\left(H-H_{1}\right) H_{1}^{\prime}\right]^{-1-j} \\
& \partial_{H}^{m_{1}}\left[1+I_{0}^{\prime}\left(H-H_{1}\right) H_{1}^{\prime}\right] \cdots \partial_{H}^{m_{j}}\left[1+I_{0}^{\prime}\left(H-H_{1}\right) H_{1}^{\prime}\right],
\end{aligned}
$$

where $0 \leq j \leq m, m_{1}+\cdots+m_{j}=m$. The proof of (3.10) follows from (3.9) and Lemma 2.4.

Case 3: $k \geq 0, l \geq 1$. Similar to the above, the proof of Case 3 is reduced to that of

$$
\left|\partial_{H}^{k} \partial_{t}^{l} I(t, H, \theta)\right| \leq C H^{-k} I(t, H, \theta)
$$

for all $k \geq 0, l \geq 1$ and $H \gg 1$. In fact, according to (3.8), Remark 2.1, and Lemma 2.4, we conclude that the differentiation of $\partial_{H}^{k} I(t, H, \theta) l$ times with respect to $t$ does not increase the order of the upper bound.

\section{Proof of the Main Result}

We rewrite (3.1) more explicitly as

$$
\frac{d t}{d \theta}=I_{0}^{\prime}(H)+\partial_{H} I_{1}(t, H, \theta), \quad \frac{d H}{d \theta}=-\partial_{t} I_{1}(t, H, \theta),
$$

where, as shown in the previous two sections, the functions $I_{0}(H)$ and $I_{1}(t, H, \theta)$ for sufficiently large $H$ satisfy the following estimates:

$$
c H^{\frac{1}{2}+\frac{1}{1+\alpha}} \leq I_{0}(H) \leq C H^{\frac{1}{2}+\frac{1}{1+\alpha}},
$$




$$
\begin{aligned}
& \frac{d^{k} I_{0}(H)}{d H^{k}} \geq c H^{-k} I_{0}(H), \quad k=1,2, \\
& \left|\frac{d^{k} I_{0}(H)}{d H^{k}}\right| \leq C H^{-k} I_{0}(H), \quad 0 \leq k \leq 5, \\
& \left|\partial_{H}^{k} \partial_{t}^{l} I_{1}(t, H, \theta)\right| \leq C H^{-k-\frac{1}{2}} I_{0}(H), \quad 0 \leq k+l \leq 5 .
\end{aligned}
$$

For $H_{0}>0$, we consider the domain

$$
A_{H_{0}}=\left\{(t, H) \mid H \geq H_{0}, t \in \mathbb{S}^{1}=\mathbb{R} / \mathbb{Z}\right\}
$$

and the diffeomorphism $\Psi: A_{H_{0}} \times \mathbb{S}^{1} \rightarrow A_{H_{0}} \times \mathbb{S}^{1}$ :

$$
\lambda=I_{0}^{\prime}(H), \quad t=t, \quad \theta=\theta .
$$

Then system (4.1) under the transformation $\Psi$ becomes

$$
\frac{d \lambda}{d \theta}=f_{1}(\lambda, t, \theta), \quad \frac{d t}{d \theta}=\lambda+f_{2}(\lambda, t, \theta),
$$

where

$$
f_{1}(\lambda, t, \theta)=-I_{0}^{\prime \prime}(H) \partial_{t} I_{1}(t, H, \theta), \quad f_{2}(\lambda, t, \theta)=\partial_{H} I_{1}(t, H, \theta),
$$

with $H=H(\lambda)$ defined through the transformation $\Psi$.

To estimate $f_{1}$ and $f_{2}$, we note by (4.2)-(4.4) that

$$
c H^{\frac{1}{1+\alpha}-\frac{1}{2}} \leq I_{0}^{\prime}(H) \leq C H^{\frac{1}{1+\alpha}-\frac{1}{2}} .
$$

It follows that

$$
c \lambda^{\frac{2(1+\alpha)}{1-\alpha}} \leq H(\lambda) \leq C \lambda^{\frac{2(1+\alpha)}{1-\alpha}} .
$$

Thus $H \gg 1$ if and only if $\lambda \gg 1$. Moreover, by (4.3), (4.4), as $\lambda \gg 1$,

$$
\left|\frac{d^{k} H(\lambda)}{d \lambda^{k}}\right| \leq C \lambda^{-k} H(\lambda),
$$

and consequently,

$$
\begin{aligned}
& \left|\lambda^{k} \partial_{\lambda}^{k} \partial_{t}^{l} f_{1}(\lambda, t, \theta)\right| \leq C H^{-2-\frac{1}{2}} I_{0}(H)^{2} \leq C H^{-\frac{3 \alpha-1}{2+2 \alpha}} \leq C \lambda^{-\frac{3 \alpha-1}{1-\alpha}} \leq C \lambda^{-\sigma} \\
& \left|\lambda^{k} \partial_{\lambda}^{k} \partial_{t}^{l} f_{2}(\lambda, t, \theta)\right| \leq C H^{-1-\frac{1}{2}} I_{0}(H) \leq C H^{-\frac{\alpha}{1+\alpha}} \leq C \lambda^{-\frac{2 \alpha}{1-\alpha}} \leq C \lambda^{-\sigma},
\end{aligned}
$$

for all $0 \leq k+l \leq 4$, where $\sigma=\min \left\{\frac{3 \alpha-1}{1-\alpha}, \frac{2 \alpha}{1-\alpha}\right\}=\frac{3 \alpha-1}{1-\alpha}>0$.

Since $f_{1}$ and $f_{2}$ are sufficiently small as $\lambda \gg 1$, all solutions of (4.6) exist for $0 \leq \theta \leq 1$ when the initial values $\lambda(0)=\lambda$ are sufficiently large. Hence the Poincaré map $\Phi$ associated to (4.6) is well defined on $A_{\lambda_{0}}$ as $\lambda_{0} \gg 1$. In fact, by integrating (4.6) from $\theta=0$ to $\theta=1$, we see that $\Phi$ has the form

$$
\Phi: \quad t_{1}=t_{0}+\lambda_{0}+\Xi_{1}\left(t_{0}, \lambda_{0}\right), \quad \lambda_{1}=\lambda_{0}+\Xi_{2}\left(t_{0}, \lambda_{0}\right),
$$

where $\Xi_{1}$ and $\Xi_{2}$ satisfy the same estimates as those of $f_{1}$ and $f_{2}$, i.e.,

$$
\left|\partial_{\lambda_{0}}^{k} \partial_{t_{0}}^{l} \Xi_{i}\right| \leq C \lambda_{0}^{-\sigma}, \quad i=1,2,0 \leq k+l \leq 4
$$


According to [1], the Poincaré map $\mathcal{P}$ associated to (4.1) admits the intersection property on $A_{H_{0}}$, i.e., if $\Gamma$ is an embedded circle in $A_{H_{0}}$ homotopic to a circle $H=$ constant, then $\mathcal{P}(\Gamma) \cap \Gamma \neq \emptyset$. It follows that $\Phi$ also admits the intersection property on $A_{\lambda_{0}}$. Hence $\Phi$ satisfies all the assumptions of Moser's twist theorem [12], [14], from which we conclude that for any $\omega \gg 1$ satisfying

$$
\left|\omega-\frac{p}{q}\right| \geq c_{0}|q|^{-\frac{5}{2}}, \quad \frac{p}{q} \in \mathbb{Q},
$$

there is an invariant curve $\Gamma_{\omega}$ of $\Phi$ which is conjugated to a pure rotation of the circle with rotation number $\omega$. Tracing back to the system (2.1), $\Gamma_{\omega}$ gives rise to an invariant closed curve of the Poincaré map of (2.1) with rotation number $\frac{1}{\omega}$ which surrounds and is arbitrarily far away from the origin. Hence all solutions of (1.1) are bounded. This completes the proof of the Theorem.

In fact, also applying Aubry-mather theory [10], [13] to the Poincaré map $\Phi$, one can obtain more precise dynamics of (1.1) as follows.

Proposition Under conditions of the Theorem, there is an $\epsilon_{0}>0$ such that the following holds.

(a) For any rational number $\frac{p}{q} \in\left(0, \epsilon_{0}\right)$, (1.1) admits an unlinked periodic solution (of Birkhoff type) with period q;

(b) For any irrational number $\omega \in\left(0, \epsilon_{0}\right)$, (1.1) admits weak quasi-periodic solutions with frequency $(1, \omega)$ corresponding to an Aubry-Mather set;

(c) For any irrational number $\omega \in\left(0, \epsilon_{0}\right)$ with $\frac{1}{\omega}$ satisfying the Diophantine condition (4.7), (1.1) admits a quasi-periodic solution with frequency $(1, \omega)$.

We finally remark that the weak quasi-periodic solutions asserted by Aubry-Mather theory form a special class of almost automorphic functions generating the almost periodic ones $([15])$.

\section{Appendix}

A1. Some properties on the potential $V(x)$. Let

$$
W(x)=\frac{V(x)}{V^{\prime}(x)}= \begin{cases}\frac{x}{\alpha+1}, & \text { if } \quad x \geq 0 ; \\ \frac{1}{2} x(1-x)(1+x), & \text { if }-1<x<0 .\end{cases}
$$

Then

$$
\begin{aligned}
W^{\prime}(x) & =\left\{\begin{array}{lr}
\frac{1}{\alpha+1}, & \text { if } x>0 ; \\
\frac{1}{2}\left(1-3 x^{2}\right), & \text { if }-1<x<0,
\end{array}\right. \\
W^{\prime \prime}(x) & = \begin{cases}0, & \text { if } x>0 ; \\
-3 x, & \text { if }-1<x<0,\end{cases}
\end{aligned}
$$




$$
W^{(3)}(x)= \begin{cases}0, & \text { if } \quad x>0 \\ -3, & \text { if }-1<x<0\end{cases}
$$

and

$$
W^{(4)}(x)=0, \quad x \in \mathbb{R} \backslash\{0\} .
$$

Also, if $n \geq 1$, then

$$
V^{(n)}(x)= \begin{cases}(\alpha+1) \alpha \cdots[(\alpha+1)-(n-1)] x^{(\alpha+1)-n}, & \text { if } \quad x>0 ; \\ g_{n}(x)(1+x)^{-n-1}, & \text { if } \quad-1<x<0,\end{cases}
$$

where $g_{n}(x)$ is defined recursively by the following formulas:

$$
\left\{\begin{array}{l}
g_{1}(x)=2 x(1-x)^{-2}, \\
g_{n+1}(x)=(1+x) g_{n}^{\prime}(x)-(n+1) g_{n}(x), \quad n \geq 1 .
\end{array}\right.
$$

It is clear that there exists a constant $C=C_{n}$ such that for all $x \in(-1,0)$,

$$
\left|V^{(n)}(x)\right| \leq C(1+x)^{-n-1}
$$

A2. Some derivative formulas. Here we list some derivative formulas which are very similar to those in [3]. Let $K(x, H)$ be a smooth function and define

$$
\begin{gathered}
\mathscr{I}(H)=\int_{-\alpha_{H}}^{0} K(\xi, H) \sqrt{H-V(\xi)} d \xi \\
\mathscr{X}(I, \theta)=\int_{-\alpha_{H}}^{x(\theta, I)} K(\xi, I) \frac{d \xi}{\sqrt{H(I)-V(\xi)}} .
\end{gathered}
$$

Then

$$
\begin{aligned}
\frac{d \mathscr{I}(H)}{d H} & =\int_{-\alpha_{H}}^{0} \mathscr{H}(K)(\xi, H) \sqrt{H-V(\xi)} d \xi \\
\partial_{I} \mathscr{X}(I, \theta) & =K(x, I) \int_{-\alpha_{H}}^{x(\theta, I)} L(\xi, I) \frac{d \xi}{\sqrt{H(I)-V(\xi)}}+\int_{-\alpha_{H}}^{x(\theta, I)} \mathscr{L}(K)(\xi, I) \frac{d \xi}{\sqrt{H(I)-V(\xi)}},
\end{aligned}
$$

where

$$
\begin{aligned}
\mathscr{H}(K)(x, H) & =\frac{1}{H}\left[H \partial_{H} K(x, H)+\frac{1}{2} K(x, H)+\partial_{x}(K(x, H) W(x))\right], \\
L(x, I) & =-\frac{H^{\prime \prime}}{H^{\prime}}+\frac{H^{\prime}}{H}\left(W^{\prime}-\frac{1}{2}\right), \\
\mathscr{L}(K)(x, I) & =\frac{H^{\prime}}{H}\left(\partial_{x}(K(I, x) W(x))-\frac{1}{2} K(x, I)\right)+\partial_{I} K(x, I) .
\end{aligned}
$$

When applying (A2.1) to $I_{0}(H)$, we will obtain its derivatives of any order. 
When applying (A2.2) to $x(I, \theta), y(I, \theta)$, we particularly have

$$
\partial_{I} x(\theta, I)=\sqrt{H-V(x)} \int_{-\alpha_{H}}^{x(\theta, I)} L(\xi, I) \frac{d \xi}{\sqrt{H-V(\xi)}}+\frac{H^{\prime}}{H} W(x)
$$

and

$$
\partial_{I} y(\theta, I)=\frac{H^{\prime}}{H} \sqrt{2(H-V(x))}-V^{\prime}(x) \int_{-\alpha_{H}}^{x(\theta, I)} L(\xi, I) \frac{d \xi}{\sqrt{2(H-V(\xi))}},
$$

where $H=H_{0}(I)$.

A3. Proof of Lemma 2.2. We recall that

$$
I_{0}^{-}(H)=2 \int_{-\alpha_{H}}^{0} \sqrt{2(H-V(\xi))} d \xi .
$$

By (A2.2), differentiating the equality $n$ times with respect to $H$ yields

$$
\frac{d^{n} I_{0}^{-}(H)}{d H^{n}}=2 \sqrt{2} \int_{-\alpha_{H}}^{0} \mathscr{H}^{n}(1)(\xi, H) \sqrt{H-V(\xi)} d \xi .
$$

Using induction (see also [[3],Lemma 6.2.1] and [[8],Proposition 5.1]), we have that for every $n \geq 1$,

$$
\mathscr{H}^{n}(1)(x, H)=\frac{1}{H^{n}} P_{n}(x),
$$

where $P_{n}(x)$ is recursively defined by

$$
\left\{\begin{array}{l}
P_{1}(x)=\frac{1}{2}+W^{\prime}(x) \\
P_{n+1}(x)=\left(\frac{1}{2}-n\right) P_{n}(x)+\left(W P_{n}\right)^{\prime}(x), \quad n \geq 1
\end{array}\right.
$$

Then

$$
\frac{d^{n} I_{0}^{-}(H)}{d H^{n}}=\frac{2 \sqrt{2}}{H^{n}} \int_{-\alpha_{H}}^{0} P_{n}(\xi) \sqrt{H-V(\xi)} d \xi
$$

or

$$
H^{n-\frac{1}{2}} \frac{d^{n} I_{0}^{-}(H)}{d H^{n}}=2 \sqrt{2} \int_{-\alpha_{H}}^{0} P_{n}(\xi) \sqrt{1-\frac{V(\xi)}{H}} d \xi .
$$

Since $\alpha_{H} \rightarrow 1$ as $H \rightarrow+\infty$, we have

$$
\lim _{H \rightarrow+\infty} H^{n-\frac{1}{2}} \frac{d^{n} I_{0}^{-}(H)}{d H^{n}}=2 \sqrt{2} \int_{-1}^{0} P_{n}(\xi) d \xi .
$$

Therefore the proof of Lemma 2.2 is reduced to the computation of the integral $\int_{-1}^{0} P_{n}(x) d x$, which had be done in [8]. Since $W(0)=W(-1)=0$ and $\int_{-1}^{0} P_{1}(x) d x=\frac{1}{2}$, we have that

$$
\begin{aligned}
\int_{-1}^{0} P_{n}(x) d x & =\left(\frac{3}{2}-n\right) \int_{-1}^{0} P_{n-1}(x) d x \\
& \left.=\cdots=\left(\frac{3}{2}-n\right)\left(\frac{3}{2}-(n-1)\right) \cdots\left(\frac{3}{2}-2\right)\right) \int_{-1}^{0} P_{1}(x) d x \\
& \left.=\cdots=\left(\frac{3}{2}-n\right)\left(\frac{3}{2}-(n-1)\right) \cdots\left(\frac{3}{2}-2\right)\right) \frac{1}{2} \\
& =(-1)^{n-1} \frac{(2 n-3) ! !}{2^{n}}
\end{aligned}
$$


This proves Lemma 2.2.

A4. Proof of Lemma 2.3. We first consider the case $n=1$.

When $x \geq 0$, we re-write (A2.3) as

$$
\begin{aligned}
\partial_{I} x(\theta, I) & =\sqrt{H-V(x)} \int_{-\alpha_{H}}^{0} L(\xi, I) \frac{d \xi}{\sqrt{H-V(\xi)}} \\
& +\sqrt{H-V(x)} \int_{0}^{x} L(\xi, I) \frac{d \xi}{\sqrt{H-V(\xi)}}+\frac{H^{\prime}}{H} W(x) .
\end{aligned}
$$

Since, for $-1<x<0,-1<W^{\prime}(x)<\frac{1}{2}$ and $\left|\frac{H^{\prime \prime}}{H^{\prime}}\right|,\left|\frac{H^{\prime}}{H}\right|$ are bounded by $C I^{-1}$ for sufficiently large $I$ (Remark 2.1), then $|L| \leq C I^{-1}$. It follows from Lemma 2.2 that, for sufficiently large $I$, the absolute value of the first term on the right hand side of (A4.1) is bounded by $C I^{-1} \sqrt{H} T_{0}^{-}(H) \leq C I^{-1}$. Since, for $0 \leq \xi \leq x, 0 \leq \frac{\sqrt{H-V(x)}}{\sqrt{H-V(\xi)}} \leq 1$, the absolute value of the second term on the right hand side of (A4.1) is bounded by $C I^{-1} x$. Using Remark 2.1 and the fact that $W(x)=\frac{x}{\alpha+1}$ for $x \geq 0$, the absolute value of the third term on the right hand side of (A4.1) is also bounded above by $C I^{-1} x$. Thus the estimate of $x(\theta, I)$ in Lemma 2.3 holds when $n=1$ and $x \geq 0$.

When $-1<x<0$, we note that $-1<\frac{W(x)}{1+x}=\frac{1}{2} x(1-x)<0$ and $\left|\frac{H^{\prime}}{H}\right|$ is bounded by $C I^{-1}$ for sufficiently large $I$ (Remark 2.1). Hence the absolute value of the second term on the right hand side of $(\mathrm{A} 2.3)$ is bounded by $C I^{-1}(1+x)$. To estimate the first term on the right hand side of (A2.3), we let

$$
A(x)=\int_{-\alpha_{H}}^{x} L(\xi, I) \frac{d \xi}{\sqrt{H-V(\xi)}}, \quad B(x)=\frac{I^{-1}\left(\alpha_{H}+x\right)}{\sqrt{H-V(x)}} .
$$

We note that $A\left(-\alpha_{H}\right)=0$ and

$$
B\left(-\alpha_{H}\right)=\lim _{x \rightarrow-\alpha_{H}} \frac{I^{-1} \cdot\left(\alpha_{H}+x\right)}{\sqrt{H-V(x)}}=-2 I^{-1} \lim _{x \rightarrow-\alpha_{H}} \frac{\sqrt{H-V(x)}}{V^{\prime}(x)}=0 .
$$

Since $V^{\prime}(x)<0$ is monotonely increasing in $(-1,0)$, the mean value Theorem, implies that there exists a $\eta \in\left[-\alpha_{H}, x\right]$ such that

$$
V(x)-H=V(x)-V\left(-\alpha_{H}\right)=V^{\prime}(\eta)\left(x+\alpha_{H}\right) .
$$

Hence

$$
\left|\frac{V^{\prime}(x)\left(\alpha_{H}+x\right)}{H-V(x)}\right| \leq 1
$$

from which it easily follows that for some sufficiently large constant $C$

$$
-C I^{-1}\left[1+\frac{1}{2} \frac{V^{\prime}(x)\left(\alpha_{H}+x\right)}{H-V(x)}\right] \leq L(x, I) \leq C I^{-1}\left[1+\frac{1}{2} \frac{V^{\prime}(x)\left(\alpha_{H}+x\right)}{H-V(x)}\right],
$$

i.e.,

$$
-C B^{\prime}(x) \leq A^{\prime}(x) \leq C B^{\prime}(x)
$$


for $-\alpha_{H} \leq x \leq 0$. Integrating the above inequalities from $-\alpha_{H}$ to $x$ yields that

$$
\left|\sqrt{H-V(x)} \int_{-\alpha_{H}}^{x} L(\xi, I) \frac{d \xi}{\sqrt{H-V(\xi)}}\right| \leq C I^{-1}\left(\alpha_{H}+x\right) .
$$

Thus the estimate of $x(\theta, I)$ in Lemma 2.3 holds when $n=1$ and $-1<x<0$.

The estimate of $x(\theta, I)$ in Lemma 2.3 for general $n$ follows from similar arguments and induction.

Similarly, we obtain the desired estimate on $y(\theta, I)$.

\section{References}

[1] R. Dieckerhoff and E. Zehnder, Boundedness of solutions via the twist theorem, Ann. Scuola. Norm. Sup. Pisa Cl. Sci., (1) 14 (1987), 79-95.

[2] T. Küpper and J. You, Existence of quasiperiodic solutions and Littlewood's boundedness problem of Duffing equations with subquadratic potentials, Nonlinear Anal., 35 (1999), 549-559.

[3] M. Levi, Quasi-periodic motions in superquadratic time-periodic potentials, Comm. Math. Phys., 143 (1) (1991), 43-83.

[4] X. Li, Boundedness of solutions for Duffings equations with semilinear potentials, J. Differential Equations, 176 (2001), 248-268.

[5] J. Littlewood, Some Problems in Real and Complex Analysis, Heath, Lexington, MA., 1968.

[6] B. Liu, Boundedness for solutions of nonlinear Hill's equations with periodic forcing terms via Moser's twist theorem, J. Differential Equations, 79 (1989), 304-315.

[7] B. Liu, On Littlewood's boundedness problem for sublinear Duffing equations, Transactions of AMS, (4) 353 (2000), 1567-1585.

[8] A. Capietto, W. Dambrosio and B. Liu, On the boundedness of solutions to a nonlinear singular oscillator, Preprint.

[9] B. Liu, Quasi-periodic solutions of forced isochronous oscillators at resonance, Preprint.

[10] J. Mather, Existence of quasi-periodic orbits for twist homeomorphisms of the annulus, Topology, 21 (1982), 457-467.

[11] G. Morris, A case of boundedness of Littlewood's problem on oscillatory differential equations, Bull. Austral. Math. Soc., 14 (1976), 71-93.

[12] J. Moser, On invariant curves of area-preserving mappings of annulus, Nachr. Akad. Wiss. Gottingen Math.-Phys., kl. II (1962), 1-20.

[13] M. Pei, Aubry-Mather sets for finite-twist maps of a cylinder and semilinear Duffing equations, $J$. Differential Equations, 113 (1994), 106-127.

[14] H. Rüssman, On the existence of invariant curves of twist mappings of an annulus, Lecture Notes in Mathematics, Vol 1007, Berlin, Heidelberg, New York, Springer-verlag 1981, 677-718.

[15] Y. Yi, On almost automorphic oscillations, Fields Inst. Commun. 42 (2004), 75-99.

[16] J. You, Boundedness for solutions of superlinear Duffing's equtions via twist curves theorems, Scientia sinica, 35 (1992), 399-412.

[17] X. Yuan, Invariant tori of Duffing-type equations, J. Differential Equations, 142 (1998), 231-262. 